\title{
Wireless Smart Sensors Networks, systems, trends and its impact in environmental monitoring
}

\author{
Luis Felipe Herrera-Quintero \\ University of Alicante \\ Technology and Computer \\ Science Department \\ PO BOX 99, 03080 \\ Alicante, Spain \\ lfherrera@dtic.ua.es
}

\author{
Francisco Macia-Perez \\ University of Alicante \\ Technology and Computer \\ Science Department \\ PO BOX 99, 03080 \\ Alicante, Spain \\ pmacia@dtic.ua.es
}

\author{
Hector Ramos-Morillo \\ University of Alicante \\ Technology and Computer \\ Science Department \\ PO BOX 99, 03080 \\ Alicante, Spain \\ hramos@dtic.ua.es
}

\author{
Carlos Lago-Gonzalez \\ CUJAE \\ Systems and Engineering \\ Research Center \\ PO BOX 11500 \\ Havana, Cuba \\ clago@dtic.ua.es
}

\begin{abstract}
Wireless Smart Sensor Networks (WSSN) offers a new paradigm regards to the way that the traditional monitoring systems have been designed, all this happens due to the impactgenerating by technology in society. The deployment of Information and Communication Technologies or ICT allow us now rely with small devices that are able to integrate into a "simple element" the potential of computing, communication and monitoring systems and from here starts to move to these minidevices, the ability of intelligence that ensure the deployment of new systems focused in environmental monitoring. In this paper we present a survey of technologies employed to wireless smart sensor networks, their management systems as well as their trends and finally we presents the design and implementation of an application for monitoring environmental variables using the WSSN and virtual instrumentation on Smartphone.
\end{abstract}

Keywords Wireless Sensor Networks, TinyOS, SOA, Web services, Embedded Computting,WSN,WSSN

\section{INTRODUCTION}

In recent years, one of the emerging technologies that have had big impact on the field of research is Wireless Smart sensor Networks (WSSN) given its diversity of features and applications. In its early days were denoted by Massachusetts Institute of Technology (MIT) as one of the 10 technologies that will change the world [1]. These networks are equipped with an infrastructure that encompasses elements of monitoring, computing and communication and give to his manager that could be the government, civil, industrial and commercial sectors the ability of instrument, observe, and react to events and phenomena in a specified environment [2]. WSSN tend to expand exponentially in a way so that these small devices can be easily deployed anywhere and collect any information from the environment. Since this technology is still emerging in the social, market penetration is still begging starting, but there are a number of research groups active in joining the study and monitoring of many phenomena. In this respect, there are already centers for collecting and processing data that are sending by the WSSN, this is the case of Sensor Signal and Information Processing Center (SENSIP) [3] led by the University of Arizona in the United States. There are also research centers who are only working in this area is the case of Center for Embedded Networked Sensing (CENS) [4] led by different universities in the United States.

Parallel to this, the development of monitoring systems based on miniaturized micro solid state technology allows a large monitoring systems using Micro-Electro-Mechanical Systems (MEMS) sensors which include a type of nano scale electrical, thermal, mechanical, optical or flow, among others [5].

On the other hand, the environmental monitoring is one of the main areas of application of this technology due to its characteristics that allow the measurement of parameters in different environmental settings such as crop management, protection of forest fires, agriculture, earthquakes, active volcano, it is also possible to use macro-instruments for measuring parameters of large-scale such as landslides, atmospheric meteorology, and finally pollution studies [6] or even for planetary exploration. [7]

This paper is organized as follows: Section two outlines the background and related works associated with WSSN as well as their management systems and their trends; section three focuses on the proposal of the environmental monitoring system; section four focuses on the design and implementation of an environmental monitoring system using WSSN, virtual instrumentation and smartphones in a real scenario; section five are carried out tests on a real scenario and finally; in section six concludes this paper and points out some future directions.

\section{BACKGROUND}

Based on the fact of knowing the potential of WSSN, identifying them as the core of new applications and new developments in research, so much so that the global market expected is around more than 4500 billion dollars which will be spent in this field for the year 2029 [8], and coupled with this, its costs will be decrease significantly due to advances in the microelectronics.

Embedded sensor network technologies have reached such impact, that is being deployed across the global spectrum, so much so that there are some macro-monitoring environmental projects such as [9] for the entire European Union, as well as initiatives [10] [11] [12] to monitor the traffic in cities and its 
pollution, or in the case of coastal areas monitoring (CESN project)[13] in the behavior of tides, are some trends in environmental monitoring.

On the other hand and in terms of environmental monitoring systems, the WSSN have the requirements posed by scenario of ubiquitous computing or embedded computing, embodied by Mark Weiser, in his article entitled "Computing in the 21st century" [14]. This scenario has led to many organizations whose objectives are to introduce this vision in everyday life. According to this, the ARTEMIS European organization that is focused in the embedded devices says that over 4 billion embedded device were sold in 2006 and its global market is close to 60 billion Euros, with an annual growth rate in $14 \%$ of rates[15, 16]. Hence the new age of embedded computing tends to change the design of environmental monitoring systems and in turn, the way of its management systems.

\section{A. Technologies}

As argument [17], sensor nodes are deployed in any area so the technology for transmitting and receiving data between the nodes are wireless in nature. In this respect to physical world, one of the major wireless technologies gave the spectrum that is presented in [18], for the development of WSSN is provided by the ZigBee Alliance [19] which is a partnership of several companies working together have developed also a standard wireless networking products for low-power devices on a network. This technology is embedded in a wide range of products and applications across commercial markets, governmental, industrial, etc. ZigBee is standardized under the IEEE 802.15.4 standard which defines the various layers for interconnecting the devices.

The WSSN tends to deploy thousands of sensors that would be gathering information which will be sent for further processing. The sensors nodes itself, have a lot of minimal resources (memory, interrupts, analog digital converters, serial ports, timers, PWM channels, etc) given that, its core is conformed by a microcontroller or in other case by a microprocessor, for that reason, there are several operating system (OS) that are dedicate to manage them. In the same way, the WSSN have been evolved still more and now there are other systems which allow them to manage its gathering data through database systems embedded into the sensor and in conjunction with a principal system.

\section{B. Operating Systems in Sensing Nodes}

The TinyOS operating system is a small core, multitasking, designed specifically for sensor networks, was developed by the University of California, Berkeley. Its code is open so many communities develop on different platforms using sensor nodes. TinyOS [20] is component-based runtime environment, designed to provide support to the sensors functionalities, this OS use the NesC language [21] that is a programming language for networked embedded systems that represent a new design space for application developers. In the same line we can fin also the Mantis OS that is a multithreaded embedded operating system for smart integrated sensor network platforms for general-purpose hardware. The
MANTIS (Multimodal Networks of In-situ Sensors) [22] or MOS sensor nodes allows performing complex tasks when they are time-sensitive. Another one that has big impact is LiteOS that is an OS dedicated to sensor networks was developed by the University of Illinois [23], the particularly of this system is that use an object-oriented programming environment called LiteC is based on $\mathrm{C}++$

As regards to OS, there is a great challenge and very big opportunity to the middleware given that, there are lot embedded systems under Reduced Instruction Set Computer (RISC) and everyday we can find more operating systems, but its resources have many similarities in the hardware components, for this reason, the WSSN becomes in a new way in order to monitoring and control systems and thus in a new way to business and services.

\section{Data Management System to WSSN}

In order to manage the information that has been gathering by the WSSN, there are some systems that allows its organization such as TinyDB [24] that is a system designed and implemented among various research agencies such as MIT, the University of California at Berkeley and the research department of Intel. TinyDB is a distributed query processor that runs on each of the nodes in a sensor network.. This was designed for the platform of the motes that was done by University of California at Berkeley and is supported by the TinyOS. The principal characteristic of TinyDB is that uses techniques can provide significant reductions in power consumption on sensor devices and this parameter is a very important to the network maintenance. In same way, there is another system that was called Cougar. Cougar is a database system [25], developed by Cornell University and shares many similarities with TinyDB. In this system the user expresses a query in a high-level declarative language that extends SQL. The sensor nodes are modeled as Abstract Data Types (ADT) with functional interfaces providing access to data encapsulation. Finally is presented the SNQL that is more than one data management system so that is a language created for the networks of smart sensors (SNQL: Sensor Network Query Language) [26]. This language provides support for the consultations in a sensor network. In this system of consultation, users can control the volumes of data and the accuracy of the results by defining terms in advance, users can also change the values of these clauses in flight mode (or when the sensor node is working).

\section{Future Trends}

Based on the large volume of data that this networks are able to gather, this technology, and their approach is directed towards the services market in any field, so the serviceoriented technologies in cohesion with the WSSN starts to participate in a paradigm shift in terms of monitoring events, so much so that from a business consulting firm Gartner predicts that $80 \%$ of IT initiatives will be service-oriented and that this type emerging technologies will make a transformational benefit for information management in businesses and governments [27]. 
When the word service is mentioned, it is common to hear the term Service-Oriented Architecture (SOA) that has been heavily impacting the field of WSSN. Some of the proposals are designed to run on SOA [28] to provide a system-based services. In the same line, the work of [29] provides services for the environmental monitoring of buildings, are a further demonstration of the penetration that has been taking the paradigm in the management of SOA services. Another works in this field was conducted by [30] who makes the term SANET (Sensor Actuator Networks) to name sensors and actuators networks and in turn suggests that the system is improved by SOSANET an approach for the management of TinySOA of the services offered on these networks. In the same way [31] and [32] identify SOA as a solution for the management of sensor networks due to the complexity of nature.

There are different technologies that use the SOA paradigm, such as case of Web Services. This paradigm involves the use of several technologies such as WSDL [33], SOAP, UDDI [34][35][36] that provide more flexibility in the any platform monitoring. In this way the WSSN can offer its functionalities through services that may be published, discovered and consumed by other elements of the system in a automatic scenario. In deed with these technologies is possible to provide a standard interface for access to any wireless sensor network independent of protocols or hardware used in the WSSN. The great advantage of this environment is flexibility, because whatever sensor that is connected to the sensor network will provide a range of services and will be able to reconfigured new processes and activities to environmental control and monitoring. All this leads to the sensors is abstracted from the characteristics of low level and it will be possible to interact with it in an environment more functional.

\section{OVERVIEW OF THE PRoposal}

As we discussed in the introduction, environmental monitoring systems require robust systems that enable to gathering data by geographical areas and therefore fairly extensive WSSN are one of the most active elements for environmental monitoring. In this regard, we propose an environmental monitoring system based on three key modules (Figure 1), the first module is based on WSSN given their characteristics and potential, the second one is a centralized system for data management, and the third one is composed of remote management and visualization systems that will help to check the environmental parameters.

The first module is composed by WSSN that have platforms dedicated of instrumentation and acquisition of different variables for several environments. The second module is based on virtual instrumentation for easy compression and processing of data that is gathering by sensor nodes, this module is responsible for storage the activity generated by each of one the sensors that are in the sensors boards. Finally, for remote management and visualization system has been designed and implemented an application that works from several devices such as smartphones, PDA (Personal digital assistants), desktop PCs between others. This was thought it in this way to ensure a portability of data sensors, from whatever device hence his administrator has the possibility to check whatever parameter that are taking by WSSN.

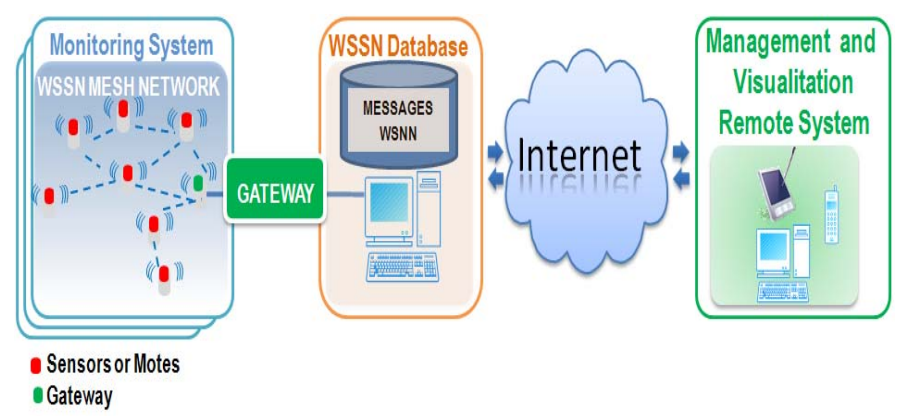

Figure 1. General scheme of the proposal.

\section{AN OVERVIEW OF THE IMPLEMENTED SYSTEM}

In this section we describe the design our environmental monitoring system. First introduce the hardware components that we have used for the development of WSSN and in second place we discuss about the infrastructure of the application, and also we describe its architecture in order to achieve an environmental monitoring system.

\section{A. Hardware Components}

In order to the deployment of WSSN we have used technology that was developed by University o Berkeley and Crossbow technologies[37], the last one, is one of the most important manufacturers of this technology. The elements that take in account the system as follows.

\section{a) Sink or Gateway}

The principal aim of the Gateway or Sink (Figure 2) is link sensor nodes and the central server where all packets are carried in transit through the WSSN. Also, these devices have the ability to manage different interfaces such as Ethernet, Wi$\mathrm{Fi}$, USB, RS232, RS485, etc, which allow the integration of WSSN in whatever field.

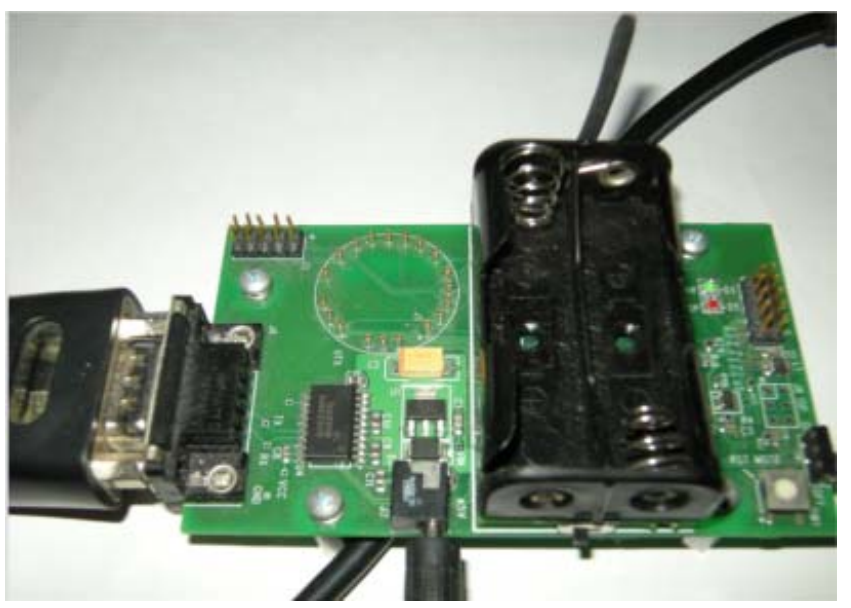

Figure 2. Gateway Crossbow Tecnologies. 


\section{b) Sensors Nodes or Motes}

The sensor nodes are micro-devices that are deployment in any area and its function is to gathering information to the central station or gateway, where it will be processed and analyzed. The devices we used are the nodes MicaZ (Figure 3) Each of ones these nodes have an ATMEL microcontroller (8bits) which includes $128 \mathrm{~K}$ Flash memory also has another parallel with $512 \mathrm{~K}$ flash memory for storing measurement of parameters and which would serve well for monitoring of environmental variables. Likewise, coming equipped with ZigBee technology through interconnection with the microcontroller circuit Chipcon 2420 which is capable of transmitting and receiving data between the nodes and from them also has a very low power and high speed bandwidth on $250 \mathrm{kbps}$, the normal frequency which this technologic operates is $2,4 \mathrm{GHz}$. The power system of each one sensors use a pair of batteries of 1.5 volts and depending on the battery amperage, the sensors nodes is able to provide adequate powering for a maximum time of 17 months, using a battery that support $3000 \mathrm{~mA}-\mathrm{Hr}$ and also offers through sleep mode $15 \mathrm{uA}$ consumption. Besides, the motes has a 51-pin connector for expansion and direct connection of sensors platforms, this connector offers direct connection to the digital analog converters (10 bit) microcontroller.

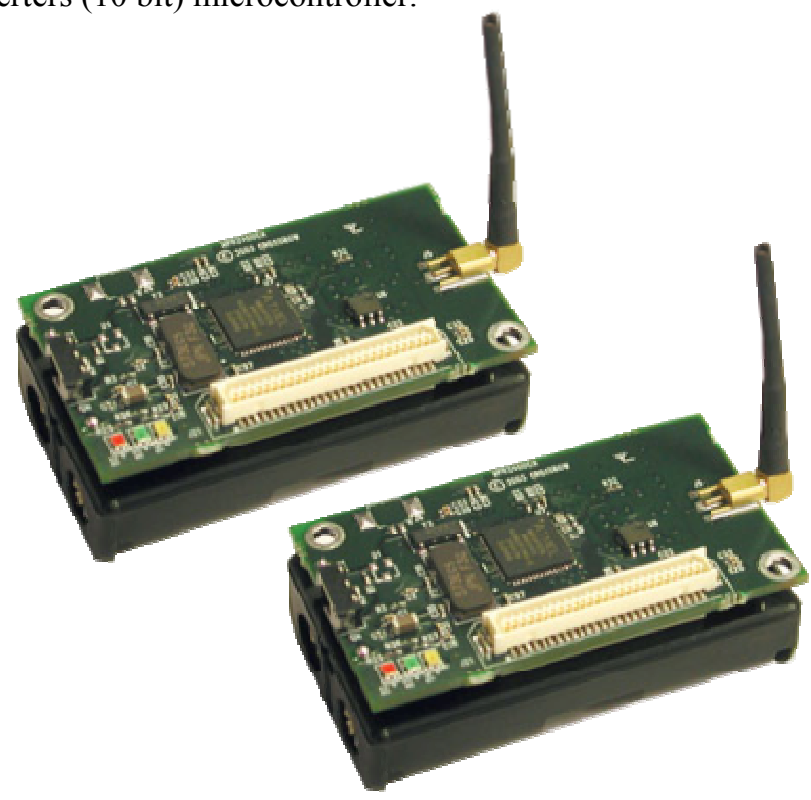

Figure 3. MicaZ Mote Crossbow Tecnologies.

c) Transducers to motes or instrumentation's platform

In the same line, the sensor nodes that we have used in the development of the application platform, brings a sensor platform that is possible to connect it through 51 pins connector. Each of these sensor boards (See Figure 4) has various sensors such as a light sensor, an accelerometer that indicates the position of the sensor in $\mathrm{X}$ and $\mathrm{Y}$, a thermistor, that offers the temperature levels, a Magnetometer that allows measure a magnetic fields, an acoustic sensor and finally a buzzer. These platforms are referenced with the type of MTS / MDA names depending on what the user wants also there are another platforms that includes sensors that are able of measure humidity, barometric pressure and even GPS (MTS420) parameters (Position's sensor in the network)
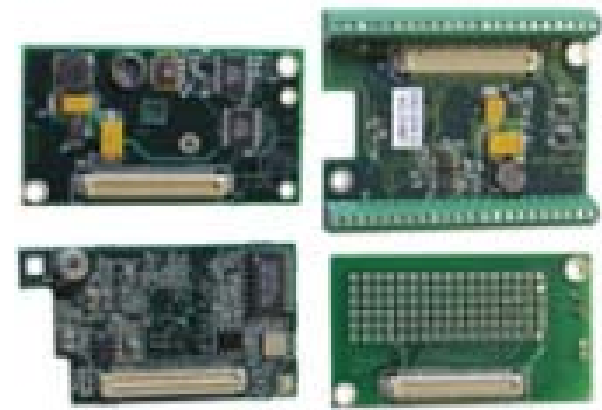

Figure 4. Sensors Boards Crossbow Technologies.

\section{B. Managament System Design}

In order to develop our application we have used the TinyOS operating system, and its programming language NesC (Figure 5). In each node runs an algorithm that manage all the sensor system and its communication with the others sensors and with the gateway. For communication between sensors and from them we have used the routing protocol called XMESH design by Crossbow [38]. This protocol is able to handling the several hopping that takes part in sensors environment and also with this protocol the nodes can send data to its base node or gateway.

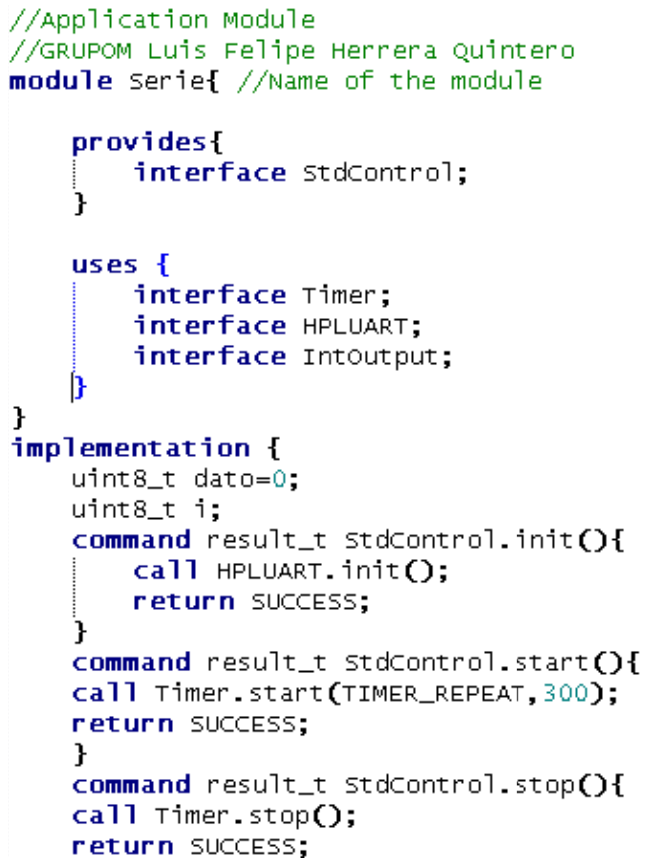

Figure 5. NesC programming Language on MICAz Motes.

To the development of management system (Figure 6) we have used a programming tool called LabVIEW [39] which receives and send data from and towards the sensor network. One of the reasons for using this tool is its main feature in the use of virtual instrumentation systems acquisition, analysis 
and visualization of data and for monitoring and control processes and more in real time.

Data from the sensor network are stored in a database which can be accessed from LabVIEW constantly and from various devices. Finally we have also designed an application for a Smartphone of last generation using virtual instrumentation based on Windows CE operating system which is able to monitor environmental variables that each of one the sensors that are present in the network and thus to predict whether there is a landslide, thanks to the accelerometer in $\mathrm{X}$ and $\mathrm{Y}$ axial position, or detecting levels of noise through acoustic sensors, or detect that the temperature at different points in the sensor network through the thermistor used in each node.

In turn, it is possible to detect the level of battery charge of the each sensor that is present into the wireless network, this characteristics is very useful as regards to procedures for maintaining the network, gives the dimensions that the WSSN could have in a large area.

To achieve the remote management and visualization system, we have used IP protocols for transmission of information. But to the future works we will use a SOA architecture that involves SOAP protocols and WSDL languages to services description. In the case of PDA or Smartphone device, we use the Wi-Fi standard communication to link the central management system and request the data are being generating by the WSSN.

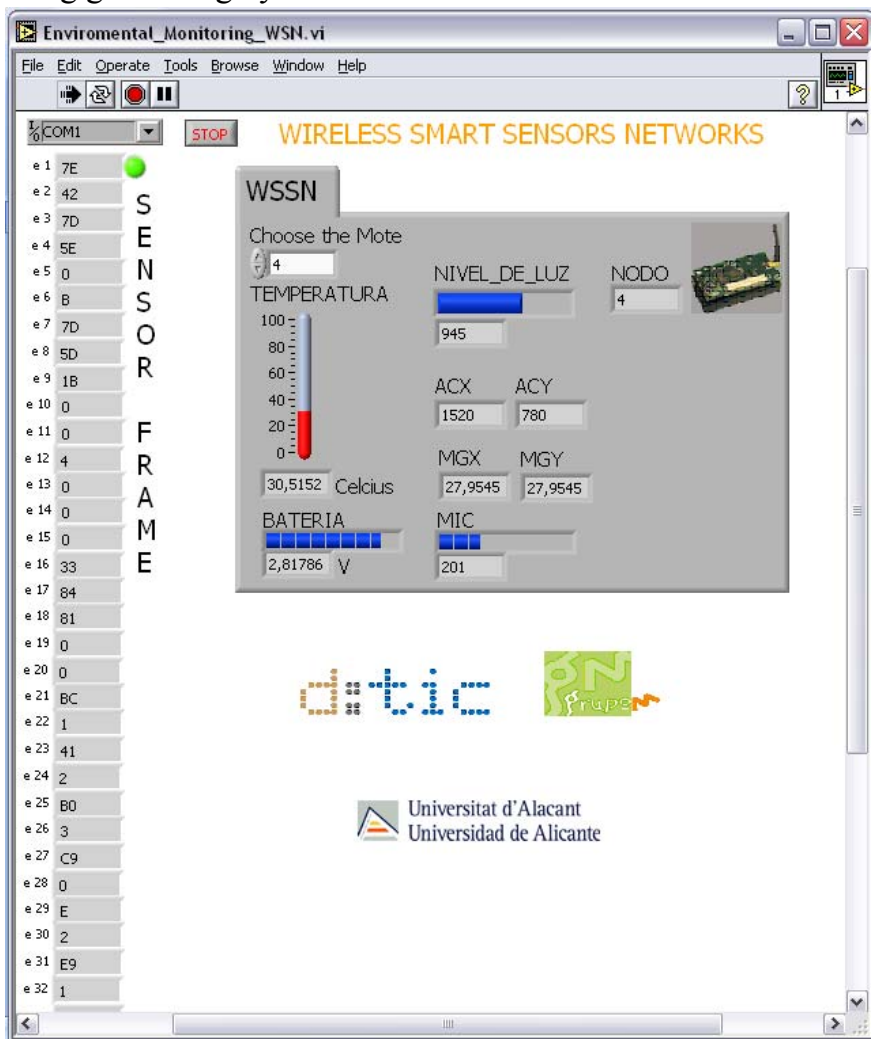

Figure 6. Magament System using LabVIEW.

\section{TEST SCENARIO}

We have used 10 sensor nodes which were deployed in a specific area of the University of Alicante, and we have measured the parameters that were gathering by the WSSN. In this way, we have used some sensors boards for environmental parameters monitoring as additional moisture and barometric pressure. We have also used a Smartphone of HTC brand (Figure 7). The application that was developed in the Smartphone used virtual instrumentation and from this device is possible remotely watch and management the behavior of each one of sensors that are present into the WSSN.

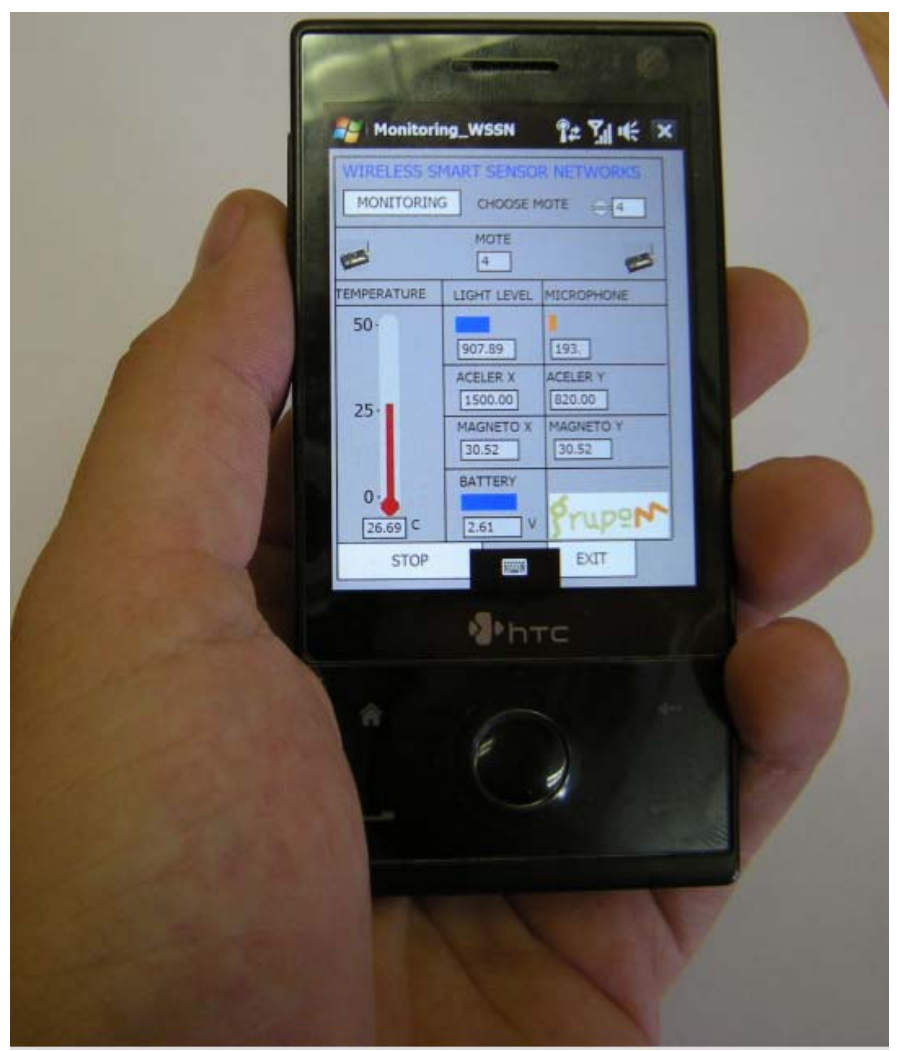

Figure 7. Remote Management and Visualization systems to WSSN

\section{CONCLUSION}

WSSN through its features such as flexibility, fault tolerance high fidelity, low cost and rapid deployment create various applications that help to establish new forms of environmental monitoring

The monitoring under IEEE 802.15.4 standard promotes the environment of WSSN that is integrated in a suitable way for monitoring and normalization.

According to future trends in WSSN need to start to deal with a scenario where service-oriented architectures SOA are involved, allowing the sensor to provide its functionality as services.

WSSN are one means of gathering data more conducive to research and to variety of scenarios make it possible to monitor both static and dynamic. If deployed, large networks of WSSN for various settings of interaction may offer larger civil service 
demand which promotes the creation of new business models based on IT.

\section{REFERENCES}

[1] Culler David, 10 Emerging Technologies that will change the world, Wireless Sensors Networks, , in MIT's Magazine of innovation technology review 2003. 2003, Massachusetts Institute of Technology: 2003. p. 36-37.

[2] Sohraby, K., D. Minoli, and T. Znati, Wireless Sensor Networks: Technology, Protocols, and Applications. 2007: Willey.

[3] SenSip. Sensor Signal \& Information Processing 2007 [cited 2008 mayo 10 de 2008]; Available from: http://enpub.fulton.asu.edu/sensip/

[4] CENS. Center for Embedded Networked Sensing. 2002 [cited Mayo 2008]; Available from: http://research.cens.ucla.edu/.

[5] Morris, C.J., S.A. Stauth, and B.A. Parviz, Self-assembly for microscale and nanoscale packaging: Steps toward self-packaging. Ieee Transactions on Advanced Packaging, 2005. 28(4): p. 600-611.

[6] Eren, H., A. Al-Ghamdi, and J. Luo. Application of ZigBee for pollution monitoring caused by automobile exhaust Gases. in SAS 2009 - IEEE Sensors Applications Symposium Proceedings. 2009.

[7] Akyildiz, I.F., et al., Wireless sensor networks: a survey. Computer Networks-the International Journal of Computer and Telecommunications Networking, 2002. 38(4): p. 393-422.

[8] Harrop, P. and R. Das, The New Market for Ubiquitous Sensor Networks (USN), IDTechEX, Editor. 2009.

[9] GMES. GMES: Global Monitoring Enviromental and Security. 2008 [cited 2008 june 10]; Available from: http://www.gmes.info/.

[10] TRACKSS. TRACKSS - Tecnologies for road advanced cooperative knowledge sharing sensors 2006 [cited 2008 May 4]; Available from: http://www.trackss.net/index.html.

[11] EMMA. EMMA - Embedded Middleware in Mobility Applications. 2006 [cited 2008 june 5]; Available from: http://www.emmaproject.eu/index.php?option=com_content\&task=view $\& \mathrm{id}=25 \&$ Itemid $=40$.

[12] AWARE. Platform for Autonomous self-deploying and operation of Wireless sensor-actuator networks cooperating with AeRial objEcts. 2006 [cited 2009 March]; Available from: http://www.awareproject.net/

[13] CESN. Coastal Environmental Sensing Networks. 2007 [cited 2007 June]; Available from: http://www.cesn.org/team.php.

[14] Weiser, M., THE COMPUTER FOR THE 21ST-CENTURY. Scientific American, 1991. 265(3): p. 94-\&.

[15] ARTEMIS. Advanced Research \& Technology for EMbedded Intelligence and Systems. 2009 [cited 2009 February]; Available from: http://www.artemis.eu/.

[16] ARTEMIS-JU. ARTEMIS Embedded Computing Systems Initiative. 2009 [cited 2009 May]; Available from: https://www.artemis-ju.eu/.

[17] Hill, J., et al., System architecture directions for networked sensors. Acm Sigplan Notices, 2000. 35(11): p. 93-104.

[18] Kuran, M.S. and T. Tugcu, A survey on emerging broadband wireless access technologies. Computer Networks, 2007. 51(11): p. 3013-3046.

[19] Zigbee, A. Zigbee Alliance. 2008 [cited 2008 Mayo 18].

[20] Levis, P., et al., TinyOS: An Operating System for Sensor Networks, in Ambient Intelligence, S.B. Heidelberg, Editor. 2005. p. 115 -148.

[21] Gay, D., et al., The nesC language: $A$ holistic approach to networked embedded systems. ACM Sigplan Notices, 2003. 38(5): p. 1-11.

[22] Bhatti, S., et al., MANTIS OS: An embedded multithreaded operating system for wireless micro sensor platforms. Mobile Networks \& Applications, 2005. 10(4): p. 563-579.

[23] Cao, Q. and T. Abdelzaher, Demo abstract: LiteOS: A lightweight operating system for $\mathrm{c}^{++}$software development in sensor networks
Proceedings of the Fourth International Conference on Embedded Networked Sensor Systems, SenSys'06:, 2006: p. 361-362.

[24] Madden, S.R., et al., TinyDB: An acquisitional query processing system for sensor networks. Acm Transactions on Database Systems, 2005. 30(1): p. 122-173.

[25] Considine, J., et al., Approximate aggregation techniques for sensor databases. 20th International Conference on Data Engineering, Proceedings, 2004: p. 449-460.

[26]. Brayner, A., et al., Toward adaptive query processing in wireless sensor networks. Signal Processing, 2007. 87(12): p. 2911-2933.

[27] Hotle M, et al., Predicts 2009: Application Governance Skills, SOA and Shifting Organizations. 2009, Gartner Consulting.

[28] Aviles-López, E. and J.A. García-Macias, TinySOA: a service-oriented architecture for wireless sensor networks. Service Oriented Computing and Applications, 2009: p. 1-10.

[29] Malatras, A., A. Asgari, and T. Baug̃̃®, Web enabled wireless sensor networks for facilities management. IEEE Systems Journal, 2008. 2(4): p. 500-512.

[30] Rezgui, A. and M. Eltoweissy, Service-oriented sensor-actuator networks [Ad hoc and sensor networks]. IEEE Communications Magazine, 2007. 45(12): p. 92-100

[31] Rezgui, A. and M. Eltoweissy, Service-oriented sensor-actuator networks [Ad hoc and sensor networks]. IEEE Communications Magazine, 2007. 45(12): p. 92-100

[32] Priyantha N, Kansal Aman, Goraczko M, Zhao F, Tiny Web Services: Design and implementation of interoperable and evolvable Sensor Networks in The 6th ACM Conference on Embedded Networked Sensor Systems (SenSys 2008) . 2008.

[33]. Prinsloo, J.M., et al. A service oriented architecture for wireless sensor and actor network applications. in ACM International Conference Proceeding Series. 2006.

[34]. Especificación WSDL del W3C. [cited 2008 May 20], Available from: http://www.w3.org/TR/wsdl.

[35]. UDDI Data Structure Refrence. [cited 2008 May 8]; Available from: http://uddi.org/pubs/DataStructure-V2.03-Published-20020719.htm.

[36].Newcomer E. Understanding Web Services - XML, WSDL. SOAP, and UDDI. Addison Wesley. 2002

[37]. Crossbow Technology. Wireless Sensor Networks [cited 2008 November]; $\quad$ Available $\quad$ from: http://www.xbow.com/Home/wHomePage.aspx

[38]. Teo, A., G. Singh, and J.C. McEachen. Evaluation of the XMesh routing protocol in wireless sensor networks. in Midwest Symposium on Circuits and Systems. 2006.

[39] National Instruments. LabVIEW [cited 2007 December] Available from: http://www.ni.com/labview/ 